\title{
Deckschichten und Bodenentwicklung in lößbedeckten Kalksenken des Sauerlandes (Rheinisches Schiefergebirge)
}

\author{
REINHOLD ROTH*)
}

Pleistocene, Weichselian, Holocene, Stratigraphy, periglacial sediments, loess, fossil soils, heavy mineral, Hemer, Rhenish massif, Northrhine-Westfalia

\begin{abstract}
Kurzfassung: Unter Lößbedeckung liegen in den Iserlohner und Attendorn-Elsper Kalksenken oft großflächig pleistozäne Fließerden, in denen außer Terrae calcis-Relikten auch Verwitterungsmaterial der umgebenden Gesteinseinheiten eingearbeitet sein kann. Mit Hilfe von Terrassenrelikten lassen sich alt- und jungpleistozäne Terra fuscaFließerden ausweisen, die sich in charakteristischer Weise unterscheiden. Örtlich begraben sie ältere Lösse mit fossiler Parabraunerdeentwicklung. Innerhalb des Weichsellösses erlauben Schwermineralanalysen stellenweise eine weitere Deckschichtengliederung und die Identifizierung der jungtundrenzeitlichen Hauptlage. Synthetische Eisensilikate weisen auf holozäne Umlagerungen und Verhüttungstätigkeiten bei Hemer hin.
\end{abstract}

\section{[Cover Sediments and Soil Development in Loess Covered Limestone Areas of the Sauerland (Rhenish Massif)]}

\begin{abstract}
Under the loess cover of the limestone areas of Iserlohn and Attendorn-Elspe widespread periglacial cover sediments occur which are mostly including relics of terrae calcis and weathered material from the surrounding rock formations. Terrace sediments are useful to identify earlyand latepleistocene periglacial sediment layers, which characteristically differ. Locally they cover older loesses with a fossil Parabraunerde. Within the Weichselian loess cover heavy minerals locally allowed the identification of the main solifluction layer of the Younger Tundra Period. Nearby Hemer the iron smelting since the 10th century led to a considerable enrichment of synthetic ironsilicates in the uppermost layers.
\end{abstract}

\section{Einleitung}

Die bodenkundlichen Kartierungen zur landwirtschaftlichen Standorterkundung (1:5000) und für die bodenkundliche Landesaufnahme (1:50 000) des Geologischen Landesamtes NRW erfaßten zwischen 1988 und 1991 u. a. Teilbereiche der Iserlohner Kalksenke und der Attendorn-Elsper Kalksenken im Sauerland. Dabei ergaben sich Hinweise auf mehrere Terra fusca- und Lößgenerationen sowie auf intensive periglaziale Umlagerungen der entsprechenden Substrate.

\footnotetext{
*) Anschrift des Verfassers: Dr. R. ROTH, Geologisches Landesamt Nordrhein-Westfalen, De-Greiff-Straße 195, 47803 Krefeld
}

\section{Morphologischer und geologisch-bodenkundlicher Überblick}

Die Attendorn-Elsper und Iserlohner Kalksenken sind mit durchschnittlichen Höhenlagen zwischen $+300-340$ und $+240-300 \mathrm{~m} \mathrm{NN}$ in die umgebende Landschaft eingemuldet. Ihr Untergrund besteht hauptsächlich aus mitteldevonischen Carbonatgesteinen, die als Zeugen ehemals ausgedehnter Riffkomplexe in unterschiedlicher Fazies ausgebildet sind. Meist handelt es sich um sehr reine Massenkalke, die stellenweise (z. B. Raum Grevenbrück) stark dolomitisiert sind.

Unter meist subtropisch bis tropisch humiden Klimabedingungen kam es seit dem Mesozoikum zu einer tiefreichenden Verkarstung und flächenhaften Einebnung der Kalksenken. Während die Verkarstung im Norden durch die Oberkreide-Transgression vermutlich bis ins Tertiär hinein unterbrochen wurde, konnten Verwitterung und Einebnung im Bereich der Attendorn-Elsper Kalksenken ungehindert fortschreiten. Dabei entstanden großflächig residuale Kalksteinverwitterungslehme, die als Terrae calcis-Reste (Terra fusca, vereinzelt Terra rossa) interpretiert werden (DAHM-ARENS 1978). Daneben kam es ab dem Ende des Tertiärs zur Ausbildung verschiedener Lenneterrassen (Clausen 1978: 283; KAMP VON 1972).

Die Kalksenken wurden in der letzten Kaltzeit mit Löß überweht, dessen Mächtigkeit heute allerdings nur selten über $2 \mathrm{~m}$ hinausgeht. Daraus entstanden durch Entkalkung, Verwitterung und z. T. Tonverlagerung tiefgründige Parabraunerden und Braunerden, die z. T. pseudovergleyt und erodiert sind (DAHM-ARENS 1976: 387; WIRTH 1976). In Oberhangund Kuppenlagen treten Braunerden und Rendzinen mit ihren Subtypen auf.

\section{Iserlohner Kalksenke}

Einen guten Überblick über Eigenschaften und Typologien der Böden der Iserlohner Kalksenke gibt WIRTH (1976), so daß für nähere Informationen auf diese Arbeiten verwiesen sei.

Zur Verdeutlichung der vielfältigen Beziehungen 


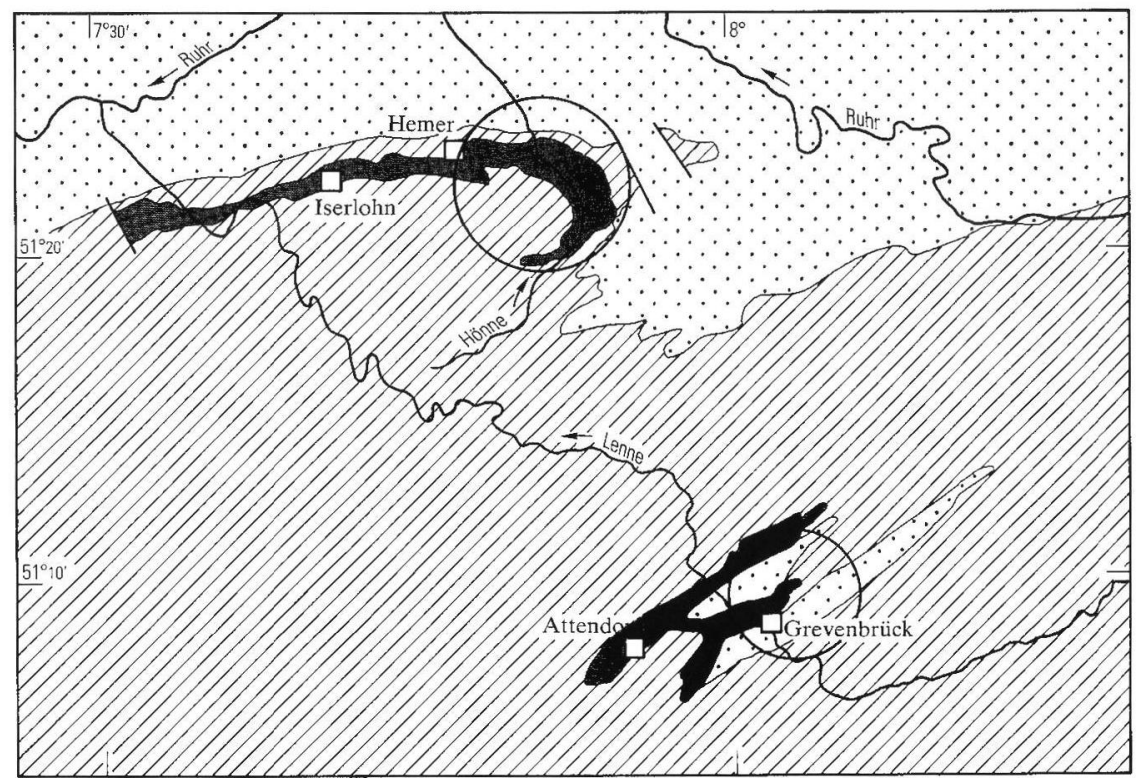

$\because \therefore$ Karbon und Perm

Massenkalk (Devoni)
Untersuchungsgebiet

0 $10 \mathrm{~km}$

Abb. 1: Geologische Übersicht und Lage der Iserlohner und Attendorn-Elsper Kalksenke

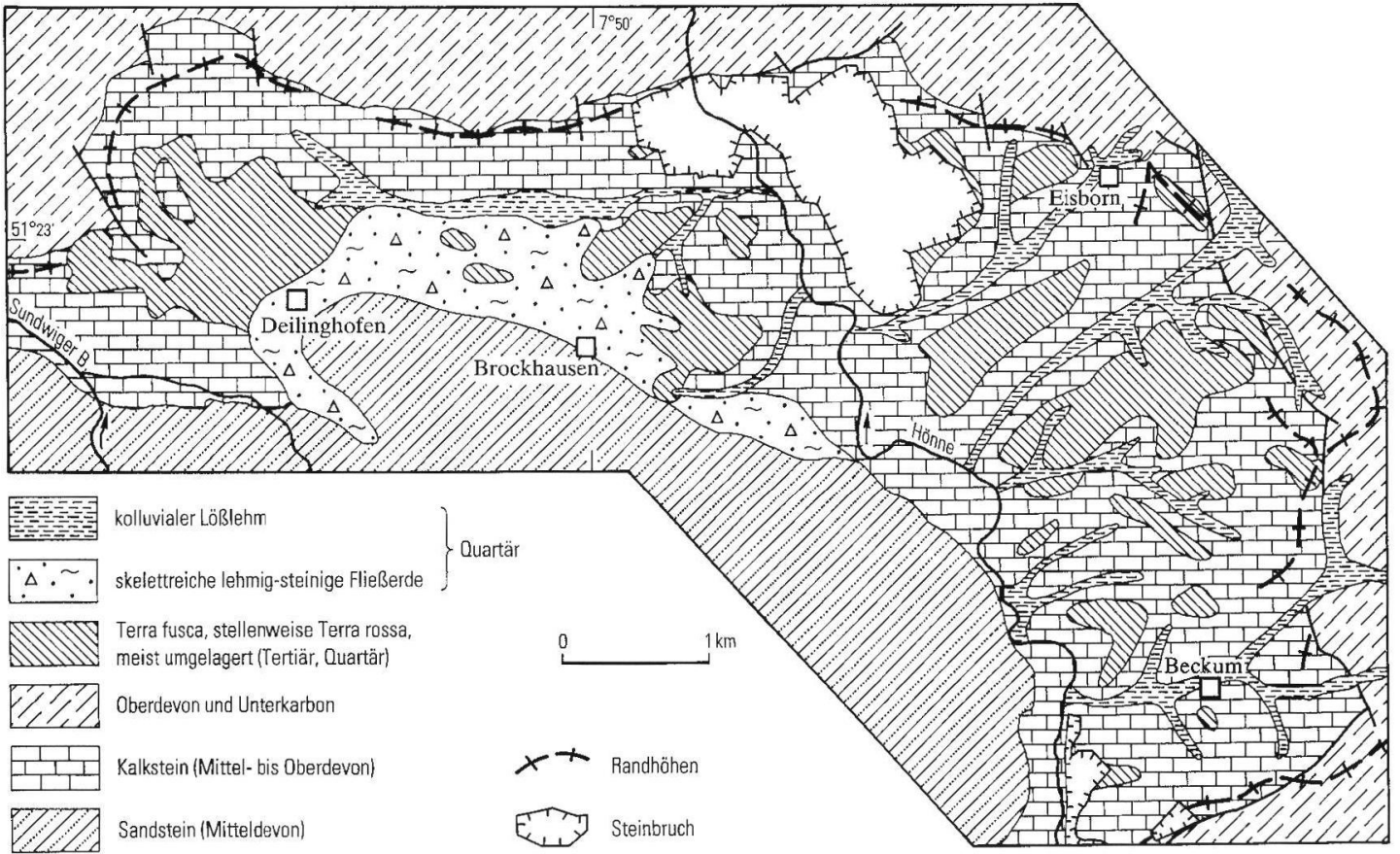

Abb. 2: Geologische Karte der Iserlohner Kalksenke zwischen Hemer und Beckum (Lößlehm entdeckt).

zwischen Morphologie, fossiler Verwitterung, Fließerdebildung, Verkarstung, Wasserhaushalt, Lößsedimentation und erosion und der Bodenent wicklung bieten sich die östlich von Hemer gelegene Deilinghofener Hochfläche und die Balver Senke an (vgl. Abb. 1). Dort erreicht der Massenkalk seine größte Ausstrichbreite und ist durch große Steinbrüche gut aufgeschlossen. Morphologisch wird die ser Bereich als Durchflußpolje gedeutet (SCHMiDT 1975: 121), das von der Hönne klammartig zer- 

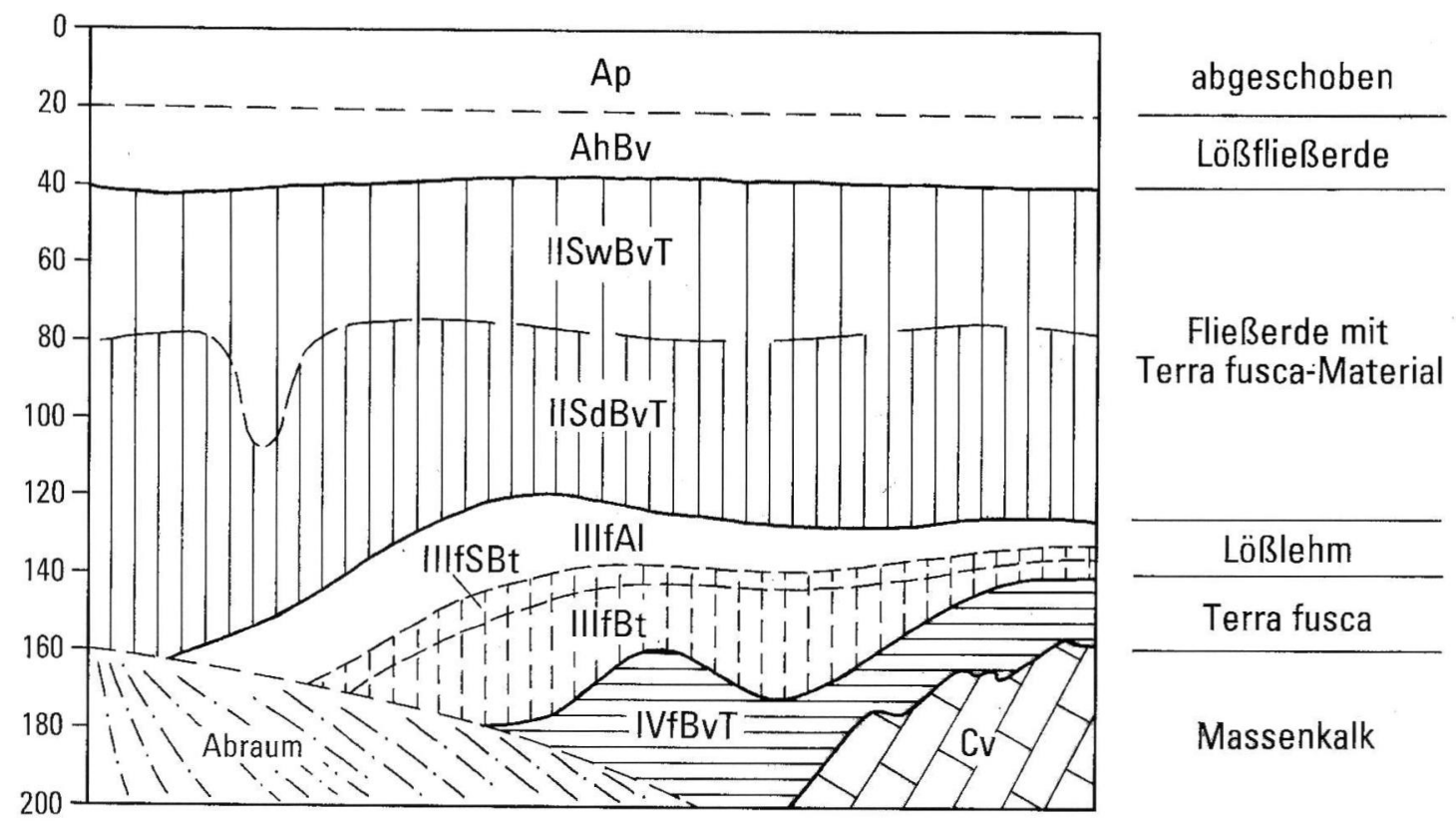

Terra fusca

Abb. 3: Fossile Pseudogley-Parabraunerde aus Lößlehm unter Terra fusca-Fließerde (Deilinghofen $\mathrm{r}^{3+} 16095, \mathrm{~h}^{56} 94230$ )

schnitten wird. In diesem Bereich werden zwei Verebnungsniveaus beschrieben, deren Verwitterungslehme sich mikromorphologisch in charakteristischer Weise unterscheiden (BURGER 1983).

\subsection{Fossile Bodenrelikte, Löß und Fließerden}

Zu den ältesten mesozoisch-tertiären Verwitterungsbildungen des Massenkalkes werden reine bunte Tone gezählt, die sich als T e r r a r os s a - Relikte deuten lassen (Clausen \& Roth in Vorb.) und sich durch sehr hohe Tongehalte (>90\%) auszeichnen. Tonärmere Hämatit und Kaolinit führende Terra rossa-Relikte werden bei Wenzens (1974:138) aus dem Felsenmeer beschrieben. Die Kartierung zeigte, daß sie nur sporadisch in erosionsgeschützten Hohlformen vorkommen und bodenbildend kaum in Erscheinung treten. Nach Burger (1983: 74) sind diese Kalkstein-Rotlehme im Bereich der Iserlohner Kalksenke an eine Verebnungsfläche $(280$ - 300 m Niveau) gebunden, die ins Oligozän gestellt wird. Eine wesentlich weitere Verbreitung nehmen dagegen braune lehmig-tonige Kalksteinverwitterungslehme ein, die im wesentlichen aus T e r r a f u s c a Relikten bestehen. Großflächig sind sie im Bereich der Verebnungsflächen erhalten geblieben, im Bereich der Täler und Randhöhen fielen sie dagegen meist der Erosion zum Opfer (vgl. Abb. 2). Die Terra fusca-Relikte sind in der Regel solifluktiv verlagert, wobei Terra rossa-Relikte, älterer Lößlehm oder ortsfremdes Sandsteinmaterial beigemischt sein können. Die so entstandenen Terra fusca-Fließerden können mehr als einen Meter mächtig werden, wobei sich stellenweise mehrere Generationen von Terra fusca-Fließerden unterscheiden lassen. Vereinzelt zeigen sie eiskeilähnliche Froststrukturen und Anzeichen einer Lessivierung (Abb. 3). Nur untergeordnet findet sich reine Terra fusca mit Tongehalten bis zu $78 \%$. Die Terra fusca-Relikte können kaolinitfrei sein (WENZENS 1974) oder aber neben Illit auch Kaolinit (möglicherweise z. T. Meta-Halloysit, Bestimmung: H. GrÜnhagen, Geol. L.-Amt Nordrh.-Westf.) enthalten.

Über den Terra fusca-Fließerden liegt auf der Südflanke der Deilinghofener Hochfläche (s. Abb. 2 und 4) eine jüngere, skelettreiche $1 \mathrm{e} \mathrm{h} \mathrm{m}$ i g-s t e i n i g e F l i e $ß$ e r d e. Diese enthält weniger Terra fusca-Material und besteht meist aus Verwitterungsmaterial der mitteldevonischen sandigen Honsel-Schichten des angrenzenden Balver Waldes. Von dort greift sie bis zu $800 \mathrm{~m}$ weit nach Norden auf die Deilinghofener Hochfläche über. Im Osten wurde diese Fließerde von der Hönne und im Westen vom Sundwiger bzw. Nieringser Bach abgeräumt (Abb. 2). Die lehmig-steinige Fließerde ist meist sehr dicht gelagert und stellt im Gegensatz zu den Terra fusca-Fließerden häufig einen Staukörper für das anfallende Sickerwasser dar.

Hinsichtlich der L Ö B a b l a g e r u n g e n waren bei den Kartierungen zwei Lößgenerationen erkennbar:

Die ältere Lößgeneration ist stellenweise unter Terra fusca-Fließerden erhalten geblieben und zeichnet sich durch hellgelbe Farbtöne aus. Der vermutlich 
ehemals kalkhaltige Löß wurde unter warmzeitlichen Bedingungen vollständig entkalkt und verwitterte zu Lößlehm. Relikte einer fossilen PseudogleyParabraunerde ließen sich in einer Baugrube bei Deilinghofen beobachten (Abb. 3).

Der jüngere weichselzeitliche Löß ist in der Iserlohner Kalksenke heute noch flächenhaft verbreitet, wobei unverwitterter kalkhaltiger Löß mit bis zu $15 \% \mathrm{CaCO}_{3}$ nur vereinzelt auftritt (ein kleines Vorkommen findet sich bei Riemke, $\mathrm{r}^{34} 18575, \mathrm{~h}^{56} 95360$ ). Meist ist der Löß vollständig entkalkt und zu Lößlehm verwittert. Auffällig ist die deutliche Asymmetrie in der Mächtigkeitsverteilung auf der Deilinghofener Hochfläche (vgl. Abb. 4). Während die Lößlehmmächtigkeiten auf der Südflanke meist unter einem Meter liegen, erreicht der Lößlehm auf der Nordflanke Mächtigkeiten bis zu zwei Metern und mehr. Dort finden sich auch ausschließlich die Vorkommen von kalkhaltigem Löß, was darauf hinweist, daß diese Mächtigkeitsunterschiede primär angelegt worden sein könnten.

Auch der Weichsellöß zeigt durch Grus- oder Steingehalte Anzeichen von Umlagerungen. Vor allem auf der Südflanke der Deilinghofener Hochfläche findet sich wieder reichlich Fremdmaterial des Balver Waldes.

\subsection{Bodengesellschaften der Deilinghofener Hochfläche und der Balver Senke}

Aus den oben beschriebenen geologischen Substraten entwickelten sich nun je nach Mächtigkeit, Lage,
Exposition und Wasserverhältnissen unterschiedliche Bodengesellschaften, wobei sich auf der Deilinghofener Hochfläche won Süden nach Norden folgende Zonen erkennen lassen (vgl. Abb. 4)

Zone der Stau- und Hangnässe-

beeinflußten Böden.

Dieser etwa 100 - 300 m breite Streifen schließt unmittelbar an den südlich bzw. südwestlich aufragenden Balver Wald an. Im tieferen Untergrund tritt zwar bereits Massenkalk auf, dieser ist jedoch durch mächtige Fließerden doppelt abgedichtet und plombiert. Die aus dem Balver Wald zuziehenden Hangwässer und Bäche können zunächst nur sehr langsam im Untergrund versickern. Es bildete sich daher weit verbreitet Pseudogley, der mit zunehmenden Lößlehmmächtigkeiten hangabwärts in PseudogleyBraunerde übergeht. Bei sehr starker Hangnässe und geringen Lößlehmmächtigkeiten kam es vereinzelt zur Bildung von Stagnogley.

Zone der überwiegenden

B ra un erden.

Hangabwärts wird die wasserstauende lehmig-steinige Fließerde geringmächtiger und wasserdurchlässiger. Stellenweise kann sie auch ganz ausfallen. Da die unterlagernden Terra fusca-Fließerden die Versickerung dagegen kaum noch behindern, kommt es zu einer zügigen Abfuhr der Sickerwässer, die durch die Verkarstung des Untergrundes zusätzlich gefördert wird. Eine besondere Rolle spielt dabei die Dolinenkette, die sich von Brockhausen
$\mathrm{S}$

\section{Balver Wald}

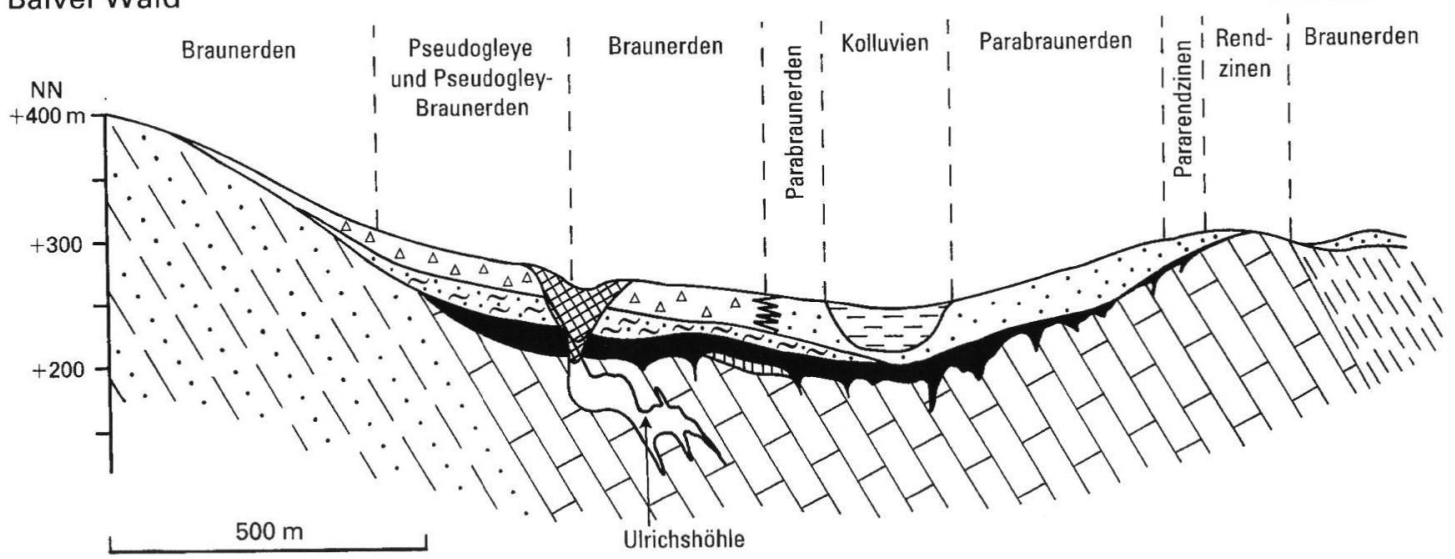
devon) 
nach Deilinghofen erstreckt. Sie wirkt zusammen mit den dazugehörigen Höhlensystemen wie ein großdimensionaler Fangdrän, der die Hangwässer abfängt und in den Untergrund leitet.

Hangabwärts überwiegen Braunerden aus Lößlehm, deren Mächtigkeit jedoch meist unter einem Meter liegt. Anzeichen für eine Parabraunerdeentwicklung mit Tonanreicherungshorizonten sind nur untergeordnet erkennbar. Kennzeichnend für diese Böden sind die Grus- und Steinanteile, die auf Umlagerungen hinweisen. Stellenweise kommen Erosionsprofile und Kolluvien vor.

Zone der überwiegenden

Parabraunerden.

Diese Zone nimmt flächenmäßig den größten Anteil ein und erstreckt sich über die Nordflanke der Deilinghofener Hochfläche bis in die Balver Senke und ist durch fruchtbare Ackerböden gekennzeichnet. Der Untergrund ist in der Regel gut durchlässig. Die Lößmächtigkeiten liegen häufig über einem Meter. Anzeichen für Tonverlagerungen und Tonanreicherungshorizonte lassen sich häufig beobachten. Bodentypologisch überwiegen Parabraunerden, die unterschiedlich stark erodiert und gekappt sein können. Abgespültes Bodenmaterial sammelte sich in Senken und Rinnen zu tiefreichend humosen Kolluvien.

Zone der Rendzinen.

Die Kalksenken werden nach Norden von Rand- höhen umrahmt, die z. T. ebenfalls noch aus Massenkalk bestehen (vgl. Abb. 2). Es herrschen Erosionsprozesse vor, wobei der Massenkalk häufig freigelegt wurde. Bodentypologisch finden sich dort Rendzinen mit allen Übergängen zu RendzinaBraunerden und Braunerden. Vereinzelt treten Pararendzinen aus kalkhaltigem Löß auf. Diese Böden sind meist ausgesprochene Trockenstandorte, so daß sie überwiegend als Wald genutzt werden.

\subsection{Schwermineralogische Untersuchungen}

Um Hinweise auf eine mögliche Deckschichtengliederung zu erhalten, wurden zwei Lößböden der Deilinghofener Hochfläche (Pseudogley-Braunerde und Parabraunerde) hinsichtlich der Schwermineralogie (Untersuchung: U. Wefels, Geol.-L.-Amt Nordrh.-Westf.) untersucht. Dabei stellte sich heraus, daß die vulkanogenen Minerale nur sehr schwach vertreten sind und eine weitere Untergliederung des weichselzeitlichen Lösses nicht ermöglichen.

Allerdings ließs sich in einer Pseudogley-Braunerde nördlich von Brockhausen $\left(\mathrm{r}^{34} 18925, \mathrm{~h}^{56} 94230\right)$ eine starke anthropogene Beeinflussung in den mittleren Bodenhorizonten nachweisen, die in der Aufgrabung mit bloßem Auge nicht erkennbar war. Dort findet sich ein synthetisches Eisensilikat (Fayalit), das bei der Eisenverhüttung entsteht (vgl. Abb. 5). Zusammen mit der schwachen Oberflächenbestreuung grünlich-glasiger Schlackenreste auf der Süd-

\section{Korngrößenverteilung}

Deckschichten/Horizonte/Tiefe (cm)

\begin{tabular}{|c|c|}
\hline holozäne & Ap \\
Oberlage & AhBv \\
& SwBv \\
\hline$?$ & Sw \\
\hline Basislage & IISd \\
\hline
\end{tabular}

Schwerminerale

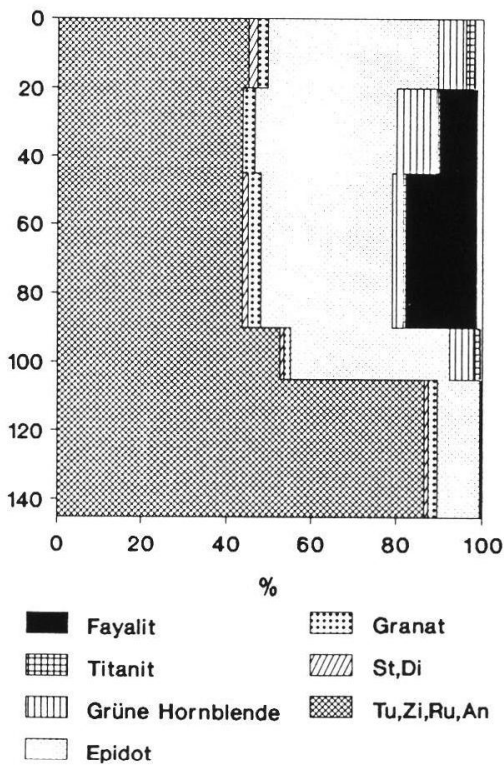

Abb. 5: Korngrößen, Schwerminerale und Deckschichtengliederung einer Pseudogley-Braunerde aus Lößlehm über lehmig-steiniger Fließerde (Brockhausen $r^{34} 30900, h^{56} 94230$ ) 
flanke der Deilinghofener Hochfläche weist dies auf eine intensive Verhüttungstätigkeit hin, deren Mindestalter im Raum Brockhausen nach HERCHENRÖDER (1967) in das 10. Jahrhundert datiert.

\section{Attendorn-Elsper Kalksenken}

Die Böden der Attendorn-Elsper Kalksenken werden bei Clausen (1978) und Dahm-Arens (1978, 1986) ausführlich beschrieben. Sie ähneln in vielerlei Hinsicht den Böden der Iserlohner Kalksenke. Ergänzende und z.T. neue Aspekte ergaben sich durch jüngere Aufgrabungen und Aufschlüsse, von denen der Dolomitsteinbruch Grevenbrück eingehender untersucht und beprobt wurde. Im folgenden wird er näher vorgestellt.

\subsection{Aufschlußbeschreibung}

Tk 25: 4814 Lennestadt; $r^{34} 30900-31050$, h ${ }^{56} 68550-$ 68650

Der aufgeschlossene Profilabschnitt (vgl. Abb. 6) erstreckt sich über einen NW-exponierten, sehr schwach geneigten Mittel- bis Oberhang, der vor der Abgrabung ackerbaulich genutzt wurde.

Den oberen Abschnitt bildet eine 50 bis $180 \mathrm{~cm}$ mächtige Decke aus kalkfreiem lehmigem bis stark lehmigem Schluff (Lößlehm). Bodentypologisch liegen im Bereich der Profile 1 und 2 Pseudogley-Parabraunerden vor, bei denen die Stauwirkung meist von den etwas dichter gelagerten Tonanreicherungshorizonten (SdBt-Horizonte) ausgeht. Diese zeigen gegenüber den Ahl- und SwAl-Horizonten
Tongehaltsdifferenzen von 5 bis 10 Gew. $\%$. Ein $(\mathrm{Sw}) \mathrm{Bv}$-Horizont ist bei diesen Böden im allgemeinen nur bei größeren Lößlehmmächtigkeiten entwickelt. Bei geringeren Mächtigkeiten haben die Vorgänge der Tonverlagerung den gesamten Lößlehm erfaßt; eine Beobachtung, die auch DAHMARENs (1986: 391) beschrieben hat. Hangaufwärts liegen gekappte Erosionsprofile (Profil 3 in Abb. 6) vor. Schleppungen an aufragenden Felsköpfen und eingelagerte Dolomitschollen weisen auf hangabwärts gerichtete Fließbewegungen hin.

Mit scharfer Grenze liegt unter dem Lößlehm eine 20-40 cm mächtige Fließerde (IIBvT- bzw. IIBtT-Horizont; Bodenfarbe meist 10 YR 3/3) aus lehmig-tonigen Terra fusca-Relikten mit Tongehalten zwischen 46 und 61 Gew.- $\%$. Die höheren Tongehalte werden dort erreicht, wo dem Verwitterungslehm (Sd)Bt-Horizonte unmittelbar aufliegen. Dort wurde ein Teil der durchschlämmten Feinsubstanz aus dem überlagernden Lößlehm bis in den Verwitterungslehm hinein transportiert.

Mit deutlicher Diskordanz folgt darunter eine Wechsellagerung von fluviatilen Sanden und kieshaltigen tonigen Fließerden (IIIBv-Horizont; Bodenfarben von dunkelbraun [7,5 YR 4/4] bis dunkelgelblichbraun [10 YR 4/6]), in denen Terra-Material aufgearbeitet ist. Den Kiesanteil stellen im wesentlichen gut gerundete Gerölle aus Gangquarz. Dieses Material ist z. T. mit den unterlagernden älteren Terra fuscaRelikten kryoturbat verwürgt, wobei sich stark ausgeprägte nordwestvergente Fließfalten ausgebildet haben (s. Abb. 6).

Die älteren Terra fusca-Relikte aus lehmigem Ton (IVBvT-Horizont) sind ebenfalls umgelagert, wie

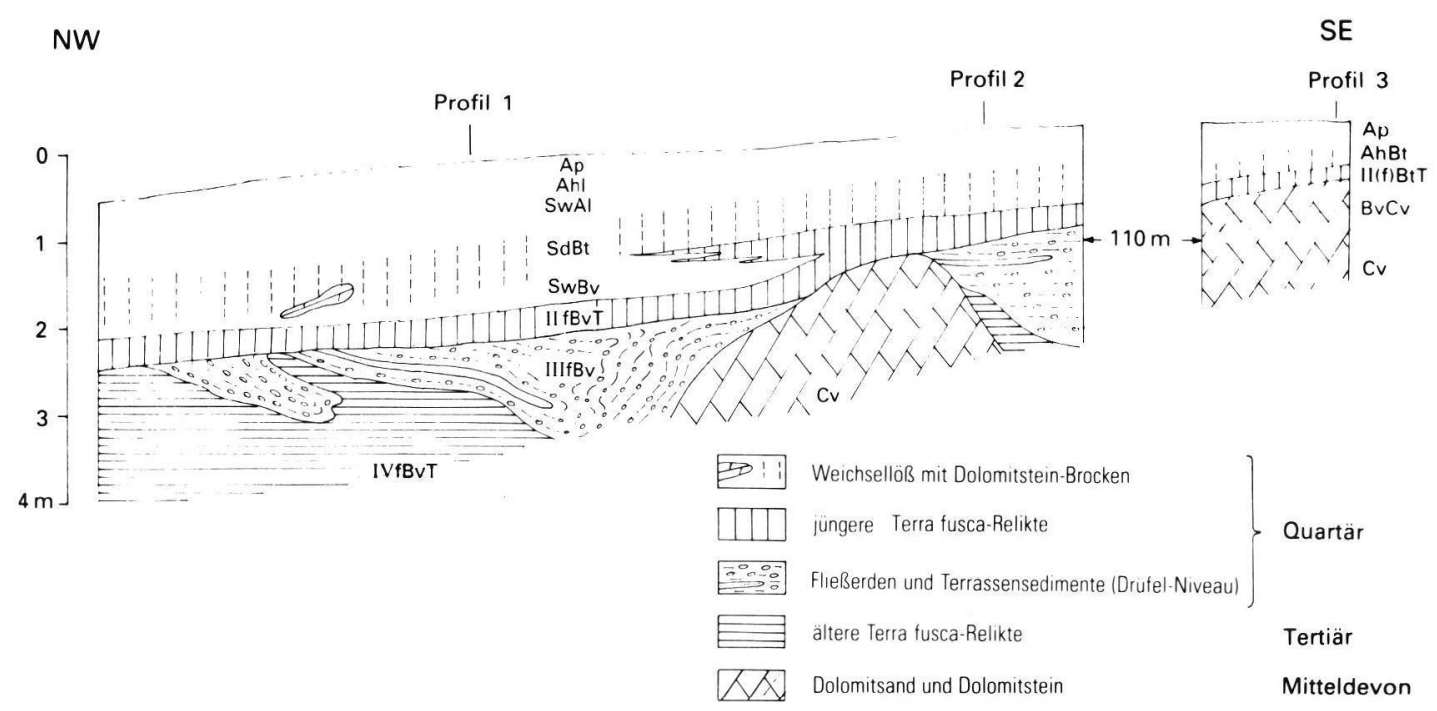


vereinzelte Quarzgerölle beweisen. Ihre Mächtigkeit kann in Schlotten mehrere Meter erreichen. Die Bodenfarben liegen zwischen dunkelgelblichbraun (10 YR 3/4) und schwerpunktmäßig dunkelbraun (7,5 YR 3/4).

Die Untergrenze zum Dolomitsand und Dolomitstein ist in der Regel scharf und deutlich ausgeprägt. Den tieferen Untergrund bildet ein massiger grauer Dolomitstein mit zuckerkörnigem Gefüge. Im Sinter enthält dieser nach Clausen (1978: 392) 57,5\% CaO und $37,0 \% \mathrm{Mg} 0$. Im oberen Bereich ist der Dolomitstein häufig zu schwach lehmigem Sand bis Sand ((Bv)Cv-Horizont) verwittert (vgl. Heilmann 1989: 59).

\subsection{Mineralogische und bodenchemische Untersuchungen}

Die Schwerminerale (Untersuchung: U. Wefels, Geol. L.-Amt Nordrh.-Westf.) erlauben im Profil 1 eine Gliederung in 3 Abschnitte (vgl. Abb. 7):

Der obere Abschnitt reicht bis etwa $100 \mathrm{~cm}$ Tiefe und umfaßt die Ap-, Ahl- und SwAl-Horizonte. Neben den lößtypischen Mineralen des Rheinspektrums (Epidot, Granat, Zirkon, Rutil u. a.) ist dieser Abschnitt durch das Auftreten der vulkanogenen Braunen Hornblende gekennzeichnet, die dort 1,5 bis $4,5 \%$ erreicht.

Der mittlere Bereich erstreckt sich über die SdBt-, SwBv- und IIBvT-Horizonte und reicht bis $180 \mathrm{~cm}$ Tiefe. Dort ähnelt das Schwermineralspektrum dem des oberen Abschnittes, wobei die Braune Hornblende jedoch stark zurücktritt. Auffällig ist ferner, daß sich die jüngeren Terra fusca-Relikte (IIBvT-Horizont) trotz der petrographisch großen Unterschiede hinsichtlich der Schwermineralführung nicht vom überlagernden Löß abheben.

Im unteren Abschnitt zeigen der fluviatile Feinsand und die älteren Terra fusca-Relikte das gleiche Schwermineralspektrum. Es zeichnet sich durch besonders hohe Anteile der stabilen Schwerminerale Zirkon, Rutil und Turmalin aus. Stellenweise können tonreichere Partien aus dem Terrassenabschnitt trotz des hohen Kiesanteils auch fast schwermineralfrei sein.

Unter den Tonmineralen dominiert bei den Terra fusca- und Terrassen-Relikten der Illit gegenüber Kaolinit. Daneben enthält die jüngere Terra fuscaFließerde noch Chlorit, der in den älteren Schichten fehlt (Untersuchung: H. Grünhagen, Geol. L-Amt Nordrh.-Westf.). Weitere Unterscheidungsmöglichkeiten zwischen jüngeren und älteren Terra fuscaFließerden ergeben sich aus der berechneten potentiellen Austauschkapazität der Tonfraktion (AKTon), die bei den jüngeren Terra fusca-Fließerden aufgrund der etwas niedrigeren Kaolinitgehalte erhöht ist. Die jüngere Terra fusca-Fließerde zeichnet sich zudem durch geringere Werte an dithionitlöslichem Eisen $\left(\mathrm{Fe}_{\mathrm{d}}\right)$ und durch höhere Aktivitätskoeffizienten $\left(\mathrm{Fe}_{\mathrm{o}} / \mathrm{Fe}_{\mathrm{d}}\right)$ aus, die absolut gesehen jedoch immer noch sehr niedrig sind (ca. 0,1). An Eisenmineralen tritt Goethit auf, Hämatit konnte nicht nach-
Korngrößenverteilung

Deckschichten/Horizonte/Tiefe (cm)

\begin{tabular}{|l|c|}
\hline Hauptlage & $\begin{array}{c}\text { Ap } \\
\text { AhI } \\
\text { SWAI }\end{array}$ \\
\hline Mittellage & SdBt \\
SwBv \\
Basislagen & $\begin{array}{r}\text { III } I I B v(T) \\
\text { IVfBVT }\end{array}$ \\
\hline
\end{tabular}
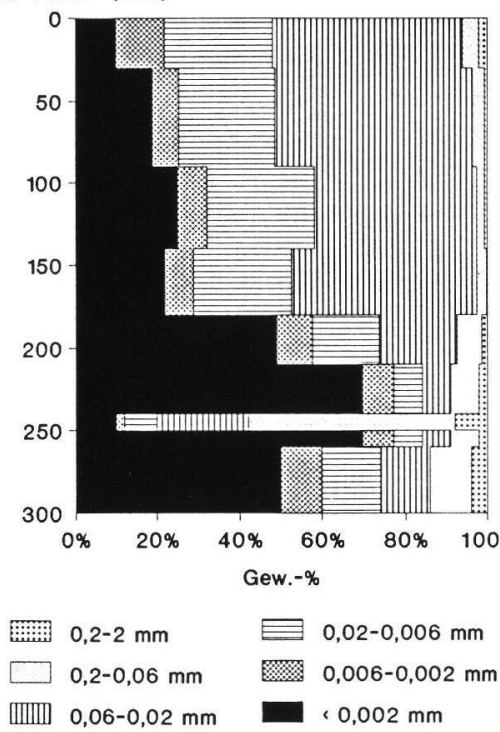

Schwerminerale

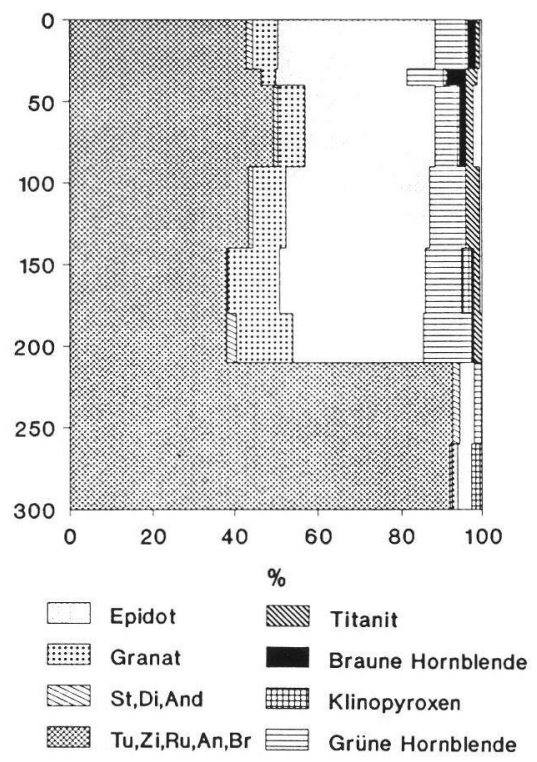

Abb. 7: Korngrößen, Schwerminerale und Deckschichtengliederung zu Profil 1 in Abb. 6 
gewiesen werden. Die $\mathrm{Fe}_{2} \mathrm{O}_{3}$-Gehalte nehmen von den jüngeren zu den älteren Terra fusca-Fließerden von 16,7 auf 27,3 Gew.- $\%$ zu.

\section{Diskussion der Ergebnisse}

\subsection{Deckschichtengliederung im Lößlehm}

Auffällig sind die Hinweise auf Umlagerungen, die sich in allen Lößggebieten des Sauerlandes beobachten lassen. Dabei stellt sich die Frage, ob die von zahlreichen Autoren aus anderen Landschaftsteilen beschriebene Gliederung der pleistozänen und holozänen Deckschichten im Sauerland wiedergefunden werden kann.

In Anlehnung an Semmel (1968), Kleber (1991), WITTMANN (1991) u. v. m. werden die pleistozänen Deckschichten in Hauptlage, Mittellage und Basislage gegliedert. Die Basislage läßt sich auch im Sauerland problemlos identifizieren. Hierbei handelt es sich in den Lößgebieten meist um die unter dem Löß liegenden Fließerden, in denen nur noch geringe Lößlehmkomponenten enthalten sind.

Eine weitere Gliederung in Haupt- und Mittellage war von den untersuchten Profilen mit einiger Sicherheit lediglich bei dem Profil Grevenbrüick mög-lich (s. Abb. 6 und 7). Dort dürfte der im oberen Bodenmeter erhöhte Anteil an Brauner Hornblende auf beigemengte allerödzeitliche Tuffkomponenten hinweisen, so daß die Ap-, Ahl- und SwAl-Horizonte der Hauptlage entsprechen, deren Bildung auf den jungtundrenzeitlichen Kälteeinbruch zurückgeführt wird.

Dies bedeutet, daßs die SwAl/SdBt-Grenze in etwa mit der Grenze Hauptlage/Mittellage zusammenfältt; eine Beobachtung, die auch in anderen Lößgebieten an Parabraunerden gemacht wurde (FRIED 1984: 82). Darüber hinaus sind im Lößlehm die Schwermineralassoziationen allerdings relativ ähnlich. Weitere Horizontunterschiede ergeben sich lediglich in dem nach unten zunehmenden Granat/Epidot-Verhältnis, das auf sekundäre Verwitterung zurückgeführt werden kann.

Weiter nördlich im Bereich Hemer konnte eine allerödzeitliche Tuffkomponente des Laacher SeeVulkanismus im Oberboden bisher nicht nachgewiesen werden.

Wie in vielen landwirtschaftlich genutzten Lößgebieten ist auch auf den Hochflächen des Sauerlandes die anthropogene Überprägung der oberen Bodenhorizonte weit intensiver als zunächst im Bohrstock erkennbar. Die Lößböden wurden im Zuge der jahrhundertelangen landwirtschaftlichen Nutzung häufig erodiert, wobei der dichtere Untergrund auf der Südflanke der Deilinghofener Hochfläche Oberflächenabfluß und Erosion erhöht haben dürfte. Ein weiterer bodenbildender Faktor war die intensive Eisenverhüttungstätigkeit südlich von Brockhausen seit dem 10. Jahrhundert; wahrscheinlich handelt es sich bei den nachgewiesenen Verhüttungsplätzen um die größten der ehemaligen Grafschaft Mark (HERCHENRÖDER 1967). Der enorme Holzbedarf führte schließlich zur Entwaldung von Teilbereichen des Balver Waldes, was auch dort den Abtrag von Bodenmaterial begünstigte, das am Hangfußs auf der Deilinghofener Hochfläche wieder abgelagert wurde. Durch dieses Nebeneinander von Bodenabtrag und Bodenauftrag kam es zur Entstehung der Fayalith führenden holozänen Oberlage. Die weite Verbreitung von Schlackenresten auf der Südflanke der Deilinghofener Hochfläche läßt zudem vermuten, daß Schlackenreste von hofeigenen Rennöfen über den Stallmist auf die Felder gebracht wurden (HERCHENRÖDER 1967).

\subsection{Terra fusca- und Fließerde-Bildung}

Terra fusca-Relikte wurden aus den Sauerländer Kalksenken schon öfter beschrieben (MEINECKE 1966, WirTH 1970, WeNZENS 1974, SCHMIDT 1975, Clausen 1978, Dahm-Akens 1978, Wirth 1982 in BrunNaCKer et al. 1982, Burger 1983 u a.), wobei für diese Bodenrelikte ein tertiäres bis quartäres Bildungsalter angenommen wird. WIRTH (in: von KAMP 1972) beobachtete zudem im Nordsauerland mehrere Terra fusca-Varietäten mit deutlichen farblichen und bodenartlichen Unterschieden, die auf unterschiedliche Bildungsbedingungen und Alter schließen lassen.

Einen ersten Hinweis auf deren Alterseinstufung gibt die tonmineralogische Zusammensetzung dieser Bodenrelikte, wobei sich die präpleistozänen TerraBöden durch hohe Kaolinit-Gehalte und die pleistozänen durch Illit-Dominanz auszeichnen (MüCKENHAUSEN \& SCHALich in: BrUNNACKER et al. 1982: 179). Ähnliche Beobachtungen macht BURGER (1983), der präoligozäne kaolinithaltige Terrae calcis-Relikte von postoligozänen kaolinitfreien Verwitterungsbildungen unterscheidet. Zeitliche Einstufungen sind jedoch angesichts der häufigen Umlagerungen und der Tatsache, daß reine Terra fusca relativ selten ist, stets mit erheblichen Unsicherheiten behaftet. Eine der wenigen datierbaren Einstufungen im nördlichen Sauerland stammt von WIRTH (1970), der für Terra fusca-Relikte ein post-altpliozänes Alter annimmt.

Im Aufschluß Grevenbrück bieten nun die mehr oder weniger umgelagerten Terrassenablagerungen weitere Datierungshilfen. Hierbei handelt es sich um Bildungen im Talrandbereich, bei denen es zu einer engen Verzahnung zwischen fluviatilen Sanden und tonigen Fließerden kam, die im wesentlichen aus aufgearbeitetem Terra-Material und Terrassenresten entstanden. Diese Ablagerungen werden aufgrund ihrer Höhenlage (65 m über Lenneniveau) der alt- 
pleistozänen Drüfel-Terrasse zugeordnet (CLAuseN 1978: 285). Für das Ausgangsmaterial der darunter liegenden altpleistozänen Terra fusca-Fließerde kann also ein tertiäres Alter angenommen werden. Kennzeichnend für die ältere Terra fusca sind neben der Färbung (7,5 YR) die höheren Kaolinit- und Eisengehalte, das Fehlen von Chlorit sowie die überwiegend stabile Schwermineralassoziation (s. o.).

Die genaue zeitliche Einstufung der jüngeren Terra fusca-Relikte, die deutlich braunere Farbtöne zeigen, läßt sich nur vermuten. Ihr relativ horizontbeständiges und flächenhaftes Auftreten - ein vergleichbares Profil wurde $500 \mathrm{~m}$ entfernt aufgegraben - und das Vorkommen an der Basis des Weichsellösses spricht für eine eemzeitliche Bildung als Kalksteinverwitterungslehm, in dem ältere Terra fuscaRelikte wahrscheinlich mit aufgearbeitet wurden (hierauf weisen die Kaolinit-Gehalte hin). Während des frühen Weichselglazials wurde dieses Material solifluktiv umgelagert, wobei es zur Vermischung mit Lößlehm kam. Dies zeigen das lößtypische Schwermineralspektrum, die günstigeren Kationenaustauschkapazitäten sowie die Chloritgehalte.

Auch im Bereich der Iserlohner Kalksenken ist eine mehrphasige Terra fusca-Bildung anzunehmen, die bis in die pleistozänen Warmzeiten hineinreichte. Dabei kam es in exponierten Kuppen und Hanglagen immer wieder zu einem Abtrag des Kalksteinverwitterungslehmes, was dazu führte, daß in Erosionslagen Terra fusca-Relikte kaum noch flächenhaft auftreten (vgl. Abb. 2). Dieser Abtrag erfolgte je nach Klima und Morphologie entweder fluviatil, wobei sich abgespültes Terra-Material in Höhlen und Dellen sammelte (Clausen \& ROTH in Vorb.) oder durch periglaziale Fließvorgänge.

Datierungen der einzelnen Fließerdegenerationen sind zur Zeit nicht möglich. Ein gewisser Hinweis für die Datierung der lehmig-steinigen Fließerde ist die Tatsache, daß sie im Bereich der linksseitigen Verebnung des Hönnetales sowie im Bereich des Sundwiger und Nieringser Baches weitgehend abgeräumt ist (s. Abb. 2). Wahrscheinlich fand dieser Abtrag statt, bevor sich die Hönne tiefer in die Hochfläche einschnitt, was für ein mindestens saalezeitliches Alter der lehmig-steinigen Fließerde spricht. Die darunter liegenden Terra fusca-Fließerden und Lößre. likte wären dementsprechend älter.

6 Schriftenverzeichnis

Brunnacker, K., Butzke, H., Dahm, H.-D., DahM-ARess, H., Dubber, H.-J., ERKWOH, F.-D., Mertens, H., Mückenhausen, E., PaAs, W., Schalich, J., Skupin, K., Will, K.-H., WIRTH, W., von ZeZschWITZ, E. (1982): Paläoböden in Nordrhein-Westfalen.- Geol. Jb., F14: 165-253, 26 Abb., 5 Tab.; Hannover.
BURGER, D. (1983): Mikromorphologische Untersuchungen der Verwitterungsresiduen im Bereich der Iserlohner Kalkmulden.- Karst und Höhle 1982/83: 73-75, 1 Abb., 1 Tab., 1 Taf.; München.

Clausex, C.-D. (1978), mit Beitr. von Fuchs, W., Gwosdz, W., Jäger, B., von Kamp, H., KreBs, W., WirTh, W., WOLF, M., Erläuterungen zu Blatt 4814 Lennestadt.- Geol. Kt. Nordrh.-Westf. 1:25000, 474 S., 29 Abb., 28 Tab.. 4 Taf.; Krefeld.

DAHM-ARENS, H. (1978): Über Reste tertiärzeitlicher Bodenbildungen im Massenkalk des südlichen Sauerlandes. Fortschr.Geol. Rheinld. u. Westf., 28: 103-110, 1 Abb.: Krefeld.

Dahm-ARens, H. (1986): Die Böden des Massenkalkes von Attendorn.- Decheniana, 139: 384-394, 3 Abb.; Bonn.

FrIED, G. (1984): Gestein, Relief und Boden im Buntsandstein-Odenwald.- Frankf.Geow.Arb., D,4: 201 S., 57 Abb., 11 Tab.; Frankfurt a. M.

Heilmann, H. (1989), mit Beitr. von Vogel, A., Exkursion A, Teil 1 Sauerland.- Mitt. Deutsche Bodenkundl. Ges., 58: 43-82, 12 Abb.; Göttingen.

HerCHENRÖDER, G. (1967): Mittelalterliche Eisenverhüttungsanlagen bei Brockhausen-Bäingsen.- Der Schlüssel, 12/4: 1-5, 2 Abb,; Hemer.

KAMP voN, H. (1972), mit Beitr. von KühN-Velten, H., SChERP, A., WIRTH, W., Wolf, M., Erläuterungen zu Blatt 4611 Hohenlimburg.- Geol. Kt. Nordrh.-Westf. 1:25000, 182 S., 18 Abb., 16 Tah., 5 Taf.; Krefeld.

KlebER, A. (1991): Gliederung und Eigenschaften der Hang-Schuttdecken und ihre Bedeutung für die Bodengenese.- Mitt. dt. bodenkdl. Ges., 66,II: 807-810; Göttingen.

Meinecke, F. (1966): Das Vorkommen von Terra rossa und Gelblehm auf dem Massenkalk im Sauerland.- Z. deutsch. Geol. Ges., 115: 715-726; Hannover.

SCHMIDT, K.-H. (1975): Geomorphologische Untersuchungen im Karstgebiet des Bergisch-Sauerländischen Gebirges. Ein Beitrag zur Tertiärmorphologie des Rheinischen Schiefergebirges.- Bochumer Geogr. Arb., 22 : 157 S., 17 Tab., 1 Kt; Paderborn.

Semmel, A. (1968): Studien über den Verlauf jungpleistozäner Formung in Hessen.- Frankfurter geogr. H., 45: 133 S., 35 Abb.; Frankfurt a. M.

WenzeNs, G. (1974): Morphogenese der Iserlohner Kalksenke.- Decheniana, 126, 1/2: 133-150; Bonn.

WIRTH, W. (1970): Eine tertiärzeitliche Karstfüllung bei Eisborn im Sauerland.- Fortschr.Geol. Rheinld. u. Westf., 17: 577-588, 6 Taf., 4 Abb., 2 Tab.; Krefeld.

WIRTH, W. (1976), mit Beitr. von KÜHN-VELTEN, H., STADLER, G., VOGLer, H., VON KAYP, H.: Erläuterungen zur Bodenkarte des Kreises und der Stadt Iserlohn 1:50000, 96 S., 9 Abb., 4 Tab.; Krefeld.

WitTMann, O. (1991): Berichte aus dem Arbeitskreis für Bodensystematik der DBG.- Mitt. Dtsch. bodenkdl. Ges., 66, I: 69-74; Göttingen.

Manuskript eingegangen am 9.11. 1992 